Mon. Not. R. Astron. Soc. 000,111() Printed 20 August $2018 \quad$ (MN LATEX style file v2.2)

\title{
Detailed cluster lensing profiles at large radii and the impact on cluster weak lensing studies
}

\author{
Masamune Oguri and Takashi Hamana \\ Division of Theoretical Astronomy, National Astronomical Observatory of Japan, 2-21-1 Osawa, Mitaka, Tokyo 181-8588, Japan.
}

20 August 2018

\begin{abstract}
Using a large set of ray-tracing in $N$-body simulations, we examine lensing profiles around massive dark haloes in detail, with a particular emphasis on the profile at around the virial radii. We compare radial convergence profiles, which are measured accurately in the ray-tracing simulations by stacking many dark haloes, with our simple analytic model predictions. Our analytic models consist of a main halo, which is modelled by the Navarro-Frenk-White (NFW) density profile with three different forms of the truncation, plus the correlated matter (2-halo term) around the main halo. We find that the smoothly truncated NFW profile best reproduces the simulated lensing profiles, out to more than 10 times the virial radius. We then use this analytic model to investigate potential biases in cluster weak lensing studies in which a single, untruncated NFW component is usually assumed in interpreting observed signals. We find that cluster masses, inferred by fitting reduced tangential shear profiles with the NFW profile, tend to be underestimated by $\sim 5-10 \%$ if fitting is performed out to $\sim 10^{\prime}-30^{\prime}$. In contrast, the concentration parameter is overestimated typically by $\sim 20 \%$ for the same fitting range. We also investigate biases in computing the signalto-noise ratio of weak lensing mass peaks, finding them to be $\lesssim 4 \%$ for significant mass peaks. In the Appendices, we provide useful formulae for the smoothly truncated NFW profile.
\end{abstract}

Key words: cosmology: theory — dark matter — galaxies: clusters: general — gravitational lensing

\section{INTRODUCTION}

Cosmological applications of clusters of galaxies rely on the inference of cluster masses. A challenge lies in the fact that clusters are dominated by dark matter, which accounts for $\sim 80 \%$ of the mass of the universe. While the distribution of dark matter in clusters can accurately be predicted using $N$-body simulations, it is difficult to relate the dark matter distribution with observable characteristics of clusters, such as gas temperatures, optical/X-ray luminosities, and member galaxy distributions, because it involves many complicated physical processes associated with gas cooling/heating and star formations. Indeed, recent cosmological constraints from X-ray analysis of clusters has mainly been limited by various astrophysical uncertainties (e.g., Vikhlinin et al. 2009; Mantz et al. 2010).

Weak gravitational lensing provides a direct probe of dark matter distributions in clusters. This method makes use of coherent tangential distortion of distant background galaxies induced by deep potential wells of clusters. For massive clusters, we can detect weak lensing signals out to virial radii, which allows direct measurements of virial masses (e.g., Dahle 2006; Broadhurst et al. 2008; Mahdavi et al. 2008; Okabe \& Umetsu 2008; Oguri et al. 2009; Umetsu et al. 2009, 2011; Medezinski et al. 2010; Okabe et al. 2010). Even for less massive clusters, one can measure an average mass of a given cluster sample by adding up lensing signals for clusters in a given sample. This stacked lensing technique has been applied to the Sloan Digital Sky Survey data to obtain constraints on cosmological parameters (Mandelbaum \& Seliak 2007; Johnston et al. 2007; Sheldon et al. 2009; Rozo et al. 2010), and will offer a promising mass-calibration method in future wide-field optical imaging surveys Oguri \& Takada 2011; Rozo, Wu, \& Schmidt 2011). In addition, stacked lensing signals much beyond virial radii probe clustering of massive haloes, which contains complementary information on halo masses (Oguri \& Takada 2011).

Weak lensing also provides a fascinating way to identify massive haloes in a way unbiased to the physical state of the baryon, by searching for peaks in the mass map reconstructed from lensing shear measurements (Schneider 1996; van Waerbeke 2000; Hamana, Takada, \& Yoshida 2004; Hennawi \& Spergel 2005; Maturi et al. 2005; Pace et al. 
2007). This idea has been applied to real data to demonstrate that massive clusters can indeed be identified (Mivazaki et al. 2002, 2007; Gavazzi \& Soucail 2007; Schirmer et al. 2007; Hamana et al. 2009; Kubo et al. 2009). Such shear-selected cluster catalogs not only offer a unique opportunity to study the relation between mass and light in a statistical manner (e.g., Geller et al. 2010), but also may provide an alternative way to constrain cosmological parameters through number counts of peaks (e.g., Kratochvil, Haiman, \& Mav 2010).

The interpretation of weak lensing data is usually made by comparing observed signals with analytical model predictions. For the analytic calculations of lensing properties, it is customary to adopt a density profile proposed by Navarro, Frenk, \& White (1997, hereafter NFW). The NFW profile has widely been used to extract information on cluster masses from weak lensing data. However, results of such analysis can be biased if the assumption on the NFW profile is not accurate. For instance, lensing signals are determined by all matter distributions along the line-of-sight, which cause the scatter and bias in mass estimates Hoekstra 2003; Dodelson 2004; de Putter \& White 2005; Marian, Smith, \& Bernstein 2010; Mandelbaum et al. 2010; Becker \& Kravtsov 2011; Hoekstra et al. 2011), which can modify lensing signals particularly at large radii. Indeed, earlier work using $N$-body simulations has found the significant contribution of correlated matter around haloes (e.g., Mandelbaum et al. 2005; Havashi \& White 2008; Tavio et al. 2008; Cacciato et al. 2009; Hilbert \& White 2010; Masaki 2011). If the true density profile deviates from the NFW profile, it can also cause a systematic bias. Understanding such bias is clearly important for attempts to use clusters as a cosmological probe.

In this paper, we investigate cluster lensing profiles in details using a large set of ray-tracing in $N$-body simulations. We pay particular attention to lensing profiles around virial radii where the origin of lensing signals should change from main haloes to the correlated matter around clusters. Thanks to the large number of ray-tracing realisations, we can study lensing profiles out to very large radii, typically several tens time virial radii of clusters. We consider an analytic model that better fit the simulated signals, which is then used to explore potential biases of several weak lensing studies originating from the assumption of the NFW profile.

The structure of this paper is as follows. In Section 2 we present analytic models adopted in the paper. Detailed comparisons with ray-tracing simulations are made in Section 3 Using the analytic model calibrated by the ray-tracing, we study potential biases in various lens studies in Section 4 We summarise the results in Section 5 In Appendices $\mathrm{A}$ and B. we provide formulae which should be useful for various calculations with the smoothly truncated NFW profile.

\section{MODELLING HALO MASS PROFILES}

\subsection{Main Halo}

In this paper, we study the radial profiles of the convergence and tangential shear around clusters. At small scales the profiles are dominated by the signals from the dark haloes associated with the clusters (the so-called 1-halo term). The current most popular model of the dark halo density profile is the profile proposed by NFW, which is defined by the following form (hereafter the NFW profile):

$\rho_{\mathrm{NFW}}(r)=\frac{\rho_{s}}{\left(r / r_{s}\right)\left(1+r / r_{s}\right)^{2}}$.

The density parameter $\rho_{s}$ is related to the virial mass $M_{\text {vir }}$ defined such that the average density within the virial radius becomes equal to the nonlinear overdensity $\Delta_{\text {vir }}$, which we compute using the spherical collapse model (see, e.g., Nakamura \& Suto 1997), times the mean matter density of the universe. Specifically, $\rho_{s}$ is described as

$\rho_{s}=\frac{\Delta_{\mathrm{vir}}(z) \bar{\rho}_{m}(z) c_{\mathrm{vir}}^{3}}{3 m_{\mathrm{nfw}}\left(c_{\mathrm{vir}}\right)}=\frac{M_{\mathrm{vir}}}{4 \pi r_{s}^{3} m_{\mathrm{nfw}}\left(c_{\mathrm{vir}}\right)}$,

where $c_{\text {vir }}$ is the so-called concentration parameter defined by

$c_{\mathrm{vir}} \equiv \frac{r_{\mathrm{vir}}}{r_{s}}=\frac{1}{r_{s}}\left[\frac{3 M_{\mathrm{vir}}}{4 \pi \Delta_{\mathrm{vir}}(z) \bar{\rho}_{m}(z)}\right]^{1 / 3}$,

and $m_{\mathrm{nfw}}\left(c_{\mathrm{vir}}\right)$ defined by

$m_{\mathrm{nfw}}\left(c_{\mathrm{vir}}\right) \equiv \int_{0}^{c_{\mathrm{vir}}} \frac{x}{(1+x)^{2}} d x=\ln \left(1+c_{\mathrm{vir}}\right)-\frac{c_{\mathrm{vir}}}{1+c_{\mathrm{vir}}}$.

The concentration parameter is known to be correlated with the halo mass and redshift. When necessary, we adopt the following relation:

$c_{\mathrm{vir}}\left(M_{\mathrm{vir}}, z\right)=7.26\left(\frac{M_{\mathrm{vir}}}{10^{12} h^{-1} M_{\odot}}\right)^{-0.086}(1+z)^{-0.71}$,

which was derived from $N$-body simulations assuming best-fit cosmological parameters in the Wilkinson Microwave Anisotropy Probe (WMAP) third year results (Macciò, Dutton, \& van den Bosch 2008), with the additional redshift dependence based on the simulation result of Duffy et al. (2008). A well-known advantage of the NFW profile in lensing studies is that there are analytic expressions for the radial profiles of the deflection angle, convergence, and shear (Bartelmann 1996; Wright \& Brainerd 2000).

However, the NFW profile is not well-defined in the sense that the enclosed mass diverges logarithmically. Thus Takada \& Jain (2003a, b) considered lensing by the NFW profile truncated at the virial radius (hereafter the TJ profile):

$\rho_{\mathrm{TJ}}(r)=\frac{\rho_{s}}{\left(r / r_{s}\right)\left(1+r / r_{s}\right)^{2}} \Theta\left(r_{\mathrm{vir}}-r\right)$,

with $\Theta(x)$ being the Heaviside step function. The lensing properties of this profile can also be computed analytically (see Takada \& Jain 2003a b).

One potential problem of the TJ profile is that the shear and convergence profiles are not differentiable at the truncation radius, which causes the divergence in the flexion profile. Baltz, Marshall, \& Oguri (2009) proposed a different form of the truncation (hereafter the BMO profile):

$\rho_{\mathrm{BMO}}(r)=\frac{\rho_{s}}{\left(r / r_{s}\right)\left(1+r / r_{s}\right)^{2}}\left(\frac{r_{t}^{2}}{r^{2}+r_{t}^{2}}\right)^{n}$,

where $r_{t}$ is the truncation radius. In the paper we mainly use the following dimensionless truncation radius 


$$
\tau_{v} \equiv \frac{r_{t}}{r_{\text {vir }}}
$$

While both $n=1$ and 2 have been considered in Baltz et al. (2009), in this paper we consider only $n=2$ for simplicity. In the case, at large radii the density profile behaves as $\rho_{\mathrm{BMO}}(r) \propto r^{-7}$, and therefore the enclosed mass converges quickly. The three-dimensional enclosed mass of the profile is

$$
\begin{aligned}
& M_{\mathrm{bmo}}(r)=4 \pi \rho_{s} r_{s}^{3} m_{\mathrm{bmo}}\left(x=r / r_{s}\right) \\
& m_{\mathrm{bmo}}(x)=\frac{\tau^{2}}{2\left(\tau^{2}+1\right)^{3}(1+x)\left(\tau^{2}+x^{2}\right)} \\
& \times\left[\left(\tau^{2}+1\right) x\left\{x(x+1)-\tau^{2}(x-1)(2+3 x)-2 \tau^{4}\right\}\right. \\
& +\tau(x+1)\left(\tau^{2}+x^{2}\right)\left\{2\left(3 \tau^{2}-1\right) \arctan (x / \tau)\right. \\
& \left.\left.+\tau\left(\tau^{2}-3\right) \ln \left(\tau^{2}(1+x)^{2} /\left(\tau^{2}+x^{2}\right)\right)\right\}\right],
\end{aligned}
$$

where $\tau \equiv r_{t} / r_{s}=\tau_{v} c_{\text {vir }}$ and we have fixed $n=2$ in equation. (77). Again, the advantage of the choice of this specific form of the truncation is that lensing properties can be computed analytically (see Baltz et al. 2009).

In equation (7), we adopted the same density parameter $\rho_{s}$ as used in the NFW profile, with $M_{\text {vir }}$ as an input parameter of this model. This virial mass $M_{\mathrm{vir}}$, strictly speaking, is different from a true virial mass defined from the requirement of the nonlinear overdensity. However, one advantage of this choice of the normalisation is that the "virial mass" of this model, $M_{\mathrm{vir}}$, becomes rather close to the total mass $M_{\text {tot }}$. From the definition above, we can show that these two masses are related by (again for $n=2$ )

$$
M_{\mathrm{tot}}=\frac{m_{\mathrm{tot}}}{m_{\mathrm{nfw}}\left(c_{\mathrm{vir}}\right)} M_{\mathrm{vir}}
$$

$$
\begin{aligned}
& m_{\mathrm{tot}} \equiv m_{\mathrm{bmo}}(\infty)=\frac{\tau^{2}}{2\left(\tau^{2}+1\right)^{3}} \\
& \times\left[\left(3 \tau^{2}-1\right)\left(\pi \tau-\tau^{2}-1\right)+2 \tau^{2}\left(\tau^{2}-3\right) \ln \tau\right] .
\end{aligned}
$$

Typically we have $M_{\text {tot }} / M_{\text {vir }} \sim 1-1.3$ and $M(<$ $\left.r_{\text {vir }}\right) / M_{\text {vir }} \sim 0.9$ in the parameter range we are interested in.

\subsection{Correlated matter}

At the large scale, the correlated matter around the cluster (the so-called 2-halo term) contributes to the lensing profile (e.g., Johnston et al. 2007). Using the Limber's approximation, we compute the 2-halo term contribution for a cluster with mass $M$ and at redshift $z$ as (e.g., Oguri \& Takada 2011)

$\kappa_{2 \mathrm{~h}}(\theta)=\int \frac{\ell d \ell}{2 \pi} J_{0}(\ell \theta) \frac{\bar{\rho}_{m}(z) b_{h}(M)}{(1+z)^{3} \Sigma_{\mathrm{crit}} D_{A}^{2}(z)} P_{m}\left(k_{\ell} ; z\right)$,

where $k_{\ell} \equiv \ell /\left\{(1+z) D_{A}(z)\right\}, D_{A}(z)$ is the angular diameter distance, $\Sigma_{\text {crit }}$ is the (physical) critical mass density for lensing, $P_{m}(k)$ is the linear matter power spectrum, and $b_{h}(M)$ is the halo bias. We can compute the corresponding radial profile of the tangential shear $\gamma_{\mathrm{T}}(\theta)$ simply by replacing the zero-th order Bessel function $J_{0}(x)$ in equation (13) to the second order Bessel function $J_{2}(x)$. The power spectrum $P_{m}(k)$ is computed using the approximation of the transfer function presented by Eisenstein \& Hu (1998).

We adopt a model of the halo bias $b_{h}(M)$ by Tinker et al. (2010), which has been calibrated using a large set of $N$-body simulations. The model has been presented as a function of several different mean overdensities $\Delta$. We adopt a model with $\Delta=800$, a larger overdensity than the virial overdensity of $\Delta \approx 300$, so that the details of the truncation near the virial radius do not affect the conversion of the masses very much. For a given set of $M_{\text {vir }}$ and $c_{\text {vir }}$, we compute the mass for $\Delta=800$ assuming the NFW profile (see, e.g., $\mathrm{Hu} \&$ Kravtsov 2003).

\section{COMPARISON WITH RAY-TRACING SIMULATIONS}

\subsection{Simulated data}

In order to study the detailed radial profiles of lensing signals around massive haloes, we employ a large set of ray-tracing simulations presented by Sato et al. (2009). The ray-tracing simulations are based on $200 \times 2$ realisations of $N$-body simulations with the box sizes of 240 (particle mass $m_{p}=$ $\left.5.4 \times 10^{10} h^{-1} M_{\odot}\right)$ and $480 h^{-1} \mathrm{Mpc}\left(m_{p}=4.4 \times 10^{11} h^{-1} M_{\odot}\right)$. The number of particles in each $N$-body run is $256^{3}$. Outputs of the small and large box simulations at different redshifts are then placed to construct a light cone of the $5^{\circ} \times 5^{\circ}$ region. We use the standard multiple lens plane algorithm to simulate lensing by intervening matter. We trace $2048^{2}$ light rays backward from the observer, resulting in the angular grid size of $0.15^{\prime}$. Thus our ray-tracing simulations take account of all density fluctuations along the line-of-sight, not just the matter distributions around massive haloes. While lens planes have been constructed out to $z=3$, in this paper we consider only one source redshift of $z_{s}=0.997$, which is a typical mean source redshift of weak lensing analysis. Thanks to the large number of $N$-body runs, 1000 independent ray-tracing realisations can finally be generated by randomly shifting the simulation boxes. The total effective area of $25000 \mathrm{deg}^{2}$ thus allows us to compute the average radial profiles quite accurately. Interested readers are referred to Sato et al. (2009) for more details of $N$-body simulations and ray-tracing technique. The assumed cosmological parameters are the matter density $\Omega_{M}=0.238$, baryon density $\Omega_{b}=0.042$, cosmological constant $\Omega_{\Lambda}=0.762$, spectral in$\operatorname{dex} n_{s}=0.958$, the normalisation of the matter fluctuation $\sigma_{8}=0.76$, and the Hubble parameter $h=0.732$, which we adopt in our analytic calculations too.

In the $N$-body simulations we identify dark haloes using the friend-of-friend (FOF) algorithm with the linking length of $b=0.2$ to derive the total mass $M_{\mathrm{FOF}}$ of each halo. We compute average convergence and tangential shear profiles in each mass and redshift bin. We consider 5 mass bins centred at $M_{\mathrm{FOF}}=10^{13.75}, 10^{14}, 10^{14.25}, 10^{14.5}$, and $10^{14.75} h^{-1} M_{\odot}$, with the bin size of $\Delta\left(\log M_{\mathrm{FOF}}\right)=0.1$, and 7 redshift bins centred at $z=0.1,0.2,0.3,0.4,0.5,0.6$, and 0.7 , with the bin size of $\Delta z=0.04$. The number of haloes used in each bin ranges from $\sim 20$ to $\sim 20000$. When stacking the profiles, we carefully choose a centre of each halo by identifying a peak in the convergence map in order to avoid 

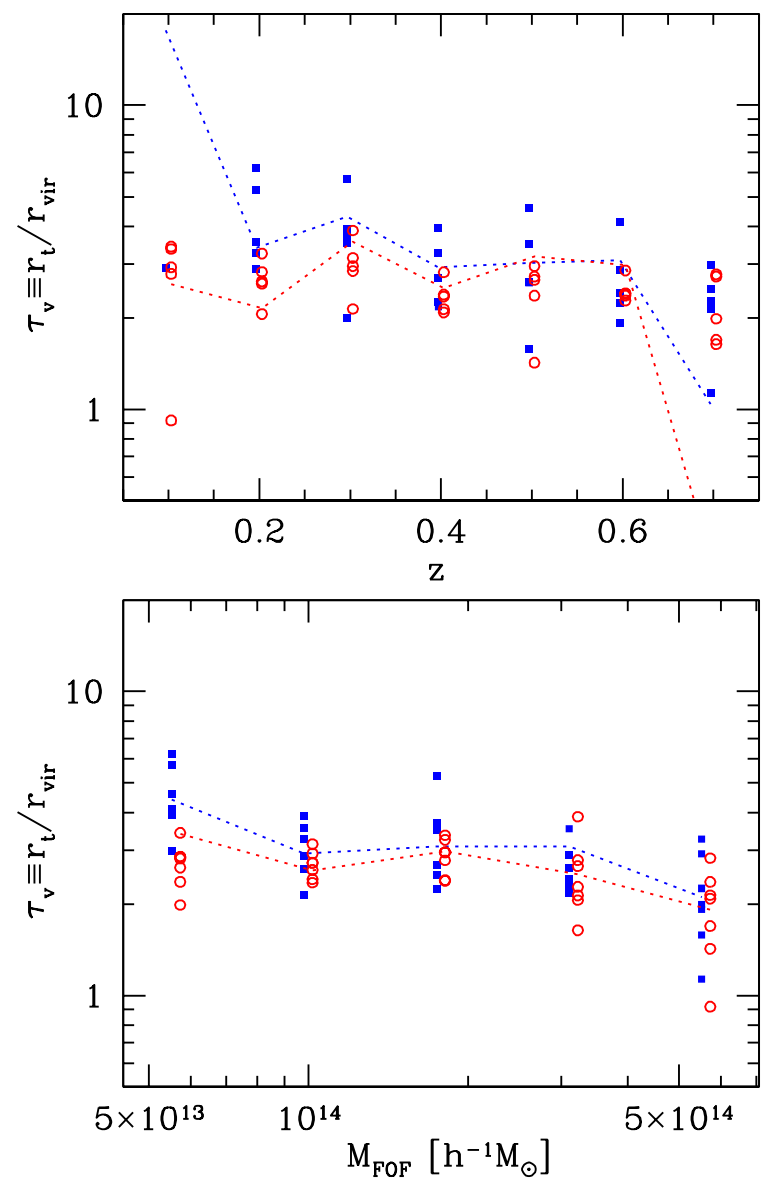

Figure 1. Bets-fit values of the dimensionless truncation radius $\tau_{v}$ (eq. [8) in the BMO profile, for different redshift (upper) and mass (lower) bins. Filled squares plot the case when the concentration parameter is computed from equation (5), whereas open circles are the case when the concentration parameter is fitted simultaneously. Dotted lines connect median values of $\tau_{v}$ in different redshift and mass bins. The median values of all bins are $\tau_{v}=3.0$ for the fixed concentration parameter, and $\tau_{v}=2.6$ for the fitted concentration parameter.

any off-centring effect which essentially smears out central profiles (see Oguri \& Takada 2011). The average profiles are computed as a function of $\theta / \theta_{\text {vir }}$, where $\theta_{\text {vir }}$ is the virial radius computed assuming $M_{\mathrm{vir}}=M_{\mathrm{FOF}}$. We derive profiles in the radius range of $10^{-2}<\theta / \theta_{\text {vir }}<50$, with the bin size of $\log \left(\theta / \theta_{\text {vir }}\right)=0.1$.

\subsection{Fitting method}

We fit the average convergence profile in each bin using the models described in Sec. 2. We basically fit the average profiles in the simulations with the sum of the 1-halo and 2-halo components, $\kappa(\theta)=\kappa_{1 \mathrm{~h}}(\theta)+\kappa_{2 \mathrm{~h}}(\theta)$, with three different models of the 1-halo components as described in Sec. 2.2 The standard $\chi^{2}$ method is employed for fitting, with the error of convergence in each radial bin derived from the scatter in the simulations. While the average profiles are derived for given ranges of $M_{\mathrm{FOF}}$, it is not clear whether $M_{\mathrm{FOF}}$ coincides with the virial mass $M_{\text {vir }}$ used in the analytic models. In fact, previous work using $N$-body simulations has found that there are systematic bias and considerable scatter between $M_{\mathrm{FOF}}$ and $M_{\mathrm{vir}}$ (e.g., White 2001; Tinker et al. 2008). Therefore, we perform fitting with leaving the virial mass $M_{\text {vir }}$ as a free parameter to take account of such systematic effect. Unless otherwise stated, we regard the concentration parameter $c_{\mathrm{vir}}$ as a free parameter, although our main conclusions are unchanged even if we fix the concentration parameter to the value obtained from equation (5). Thus there are 2 fitting parameters $\left(M_{\mathrm{vir}}, c_{\mathrm{vir}}\right)$ for the NFW and TJ profiles, and 3 fitting parameters $\left(M_{\mathrm{vir}}, c_{\mathrm{vir}}, \tau_{v}\right)$ for the BMO profile.

It has been known that very central density profiles of dark haloes in $N$-body simulations are not reliable because of several numerical effects such as the two-body relaxation and the finite time step size. Based on the detailed analysis of Fukushige \& Makino (2001), we estimate that density profiles are reliable down to $\sim 0.08 r_{\text {vir }}$ for our $N$-body simulations in which $N \sim 10^{4}$ particles are included in each halo analysed in the present paper. Indeed, our analysis results also indicate that convergence and tangential shear profiles near the very centre $\left(\theta / \theta_{\text {vir }} \sim 0.01\right)$ tend to be significantly smaller than our analytic model calculations (see below). Thus, in all mass and redshift bins fitting is performed in the radial bins $\theta / \theta_{\text {vir }} \geqslant 0.079$ to make sure that our fitting results are not affected by numerical artifacts.

\subsection{Result}

First we check the best-fit values of the truncation radius $\tau_{v}$ (eq. 8) in the BMO profile. Figure 1 show the best-fit values for different redshift and mass bins. We find the bestfit values to be $\sim 2-3$, showing no strong dependence on the mass and redshift. We consider two cases, the case that the concentration parameter is fixed to the value computed in equation (5) and the case that the concentration parameter is also fitted to the data. We find that the results of both cases agree reasonably well with each other, which implies that the best-fit values of $\tau_{v}$ is not sensitive to how the concentration parameter is treated. The median values are $\tau_{v}=3.0$ when the concentration parameter is fixed, and $\tau_{v}=2.6$ when the concentration parameter is fitted to the data.

To illustrate how well the different main halo models can reproduce the average profiles in ray-tracing simulations, in Figure 2 we show the comparisons of convergence and tangential shear profiles for a representative case. As clearly shown in the Figure, the BMO profile reproduce profiles in the ray-tracing simulations quite successfully for a wide range in radii. In contrast, the NFW and TJ profiles are less successful in fitting the profile around the virial radius, where the transition between 1-halo and 2-halo terms occurs. The NFW profile tends to overpredict the convergence profile, whereas the TJ profile clearly underpredicts the profile. For comparison, we fit the convergence profile using the 1-halo term only for the NFW and TJ profile. We find that the NFW profile can fit the total average profile quite well out to $\theta / \theta_{\text {vir }} \sim 2$, beyond which the NFW profile clearly underpredicts the profile. The situation is worse for the TJ profile for which we can see the significant discrepancy already at $\theta / \theta_{\text {vir }} \sim 1$.

The Figure also indicates that the analytic and simulated convergence profiles agree well with each other down 

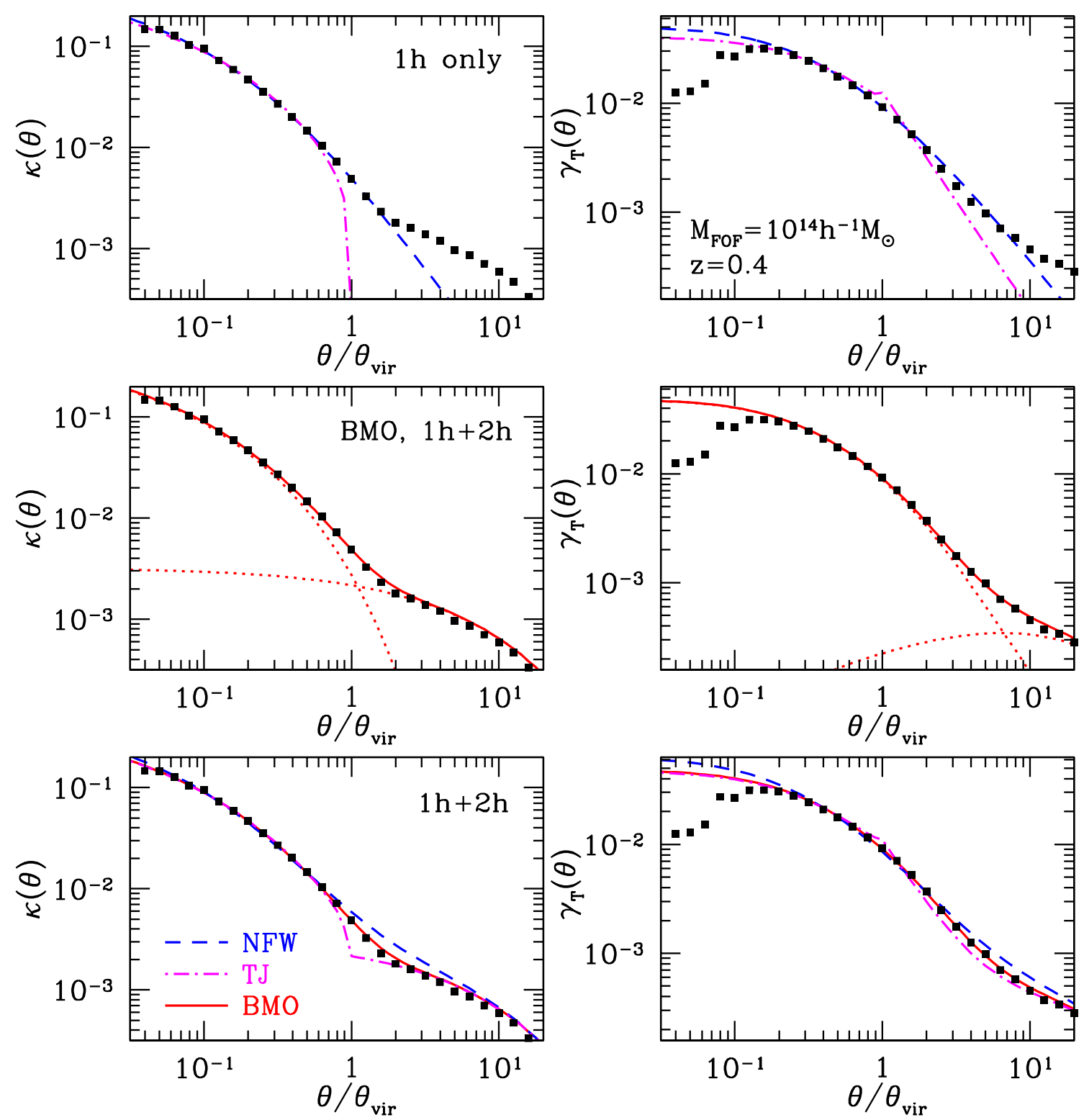

Figure 2. Example of fitting results for the mass bin $M_{\mathrm{FOF}}=10^{14} h^{-1} M_{\odot}$ and the redshift bin $z=0.4$. Left panels show convergence profiles, whereas right panels display tangential shear profiles. Curves are best-fit results for three different main halo models, the NFW profile (dashed), the TJ profile (dash-dotted), and the BMO profile (solid). The average profiles from ray-tracing simulations are indicated by filled squares. Top: Results when only 1-halo term (i.e., no 2-halo term) is considered. The result for the BMO is similar to the NFW result, and therefore not shown. Middle: Results for the BMO profile. Contributions from 1-halo and 2-halo terms are shown by dotted lines. Bottom: Results for all the 3 profiles are compared.

to very small radii of $\theta / \theta_{\text {vir }} \sim 0.05$, whereas the tangential shear profiles show large deviations already at $\theta / \theta_{\text {vir }} \sim 0.1$. One reason for this is that shear signals are non-local. Since the tangential shear signal at certain radius reflects all the mass distributions at smaller radii, numerical effects appear at larger radii in the tangential shear profiles than in the convergence profiles.

Figure 3 show residuals of the convergence profile, i.e., the fractional difference between profiles in simulations and best-fit analytic models, averaged over all redshift and mass bins. We find that the BMO profile can fit the convergence profiles in simulations quite well $(<5 \%)$ for a wide range of radii from the core of main haloes to more than 10 times the virial radii. We find the NFW profile generally overpredicts the convergence profile by $\sim 20-30 \%$ at $\theta / \theta_{\text {vir }} \sim 2$. On the other hand, the TJ profile grossly underpredicts the convergence profile at $\theta / \theta_{\text {vir }} \sim 1$. Thus we confirm the result shown in Figure 2 that the BMO profile can best describe convergence profiles in ray-tracing simulations. Again, we check residuals for the case that we include only 1-halo term in the analytic model, and find that the NFW profile can fit the convergence profiles quite well out to $\theta / \theta_{\text {vir }} \sim 1$, but begins to underpredicts the profile quickly beyond the radius. 


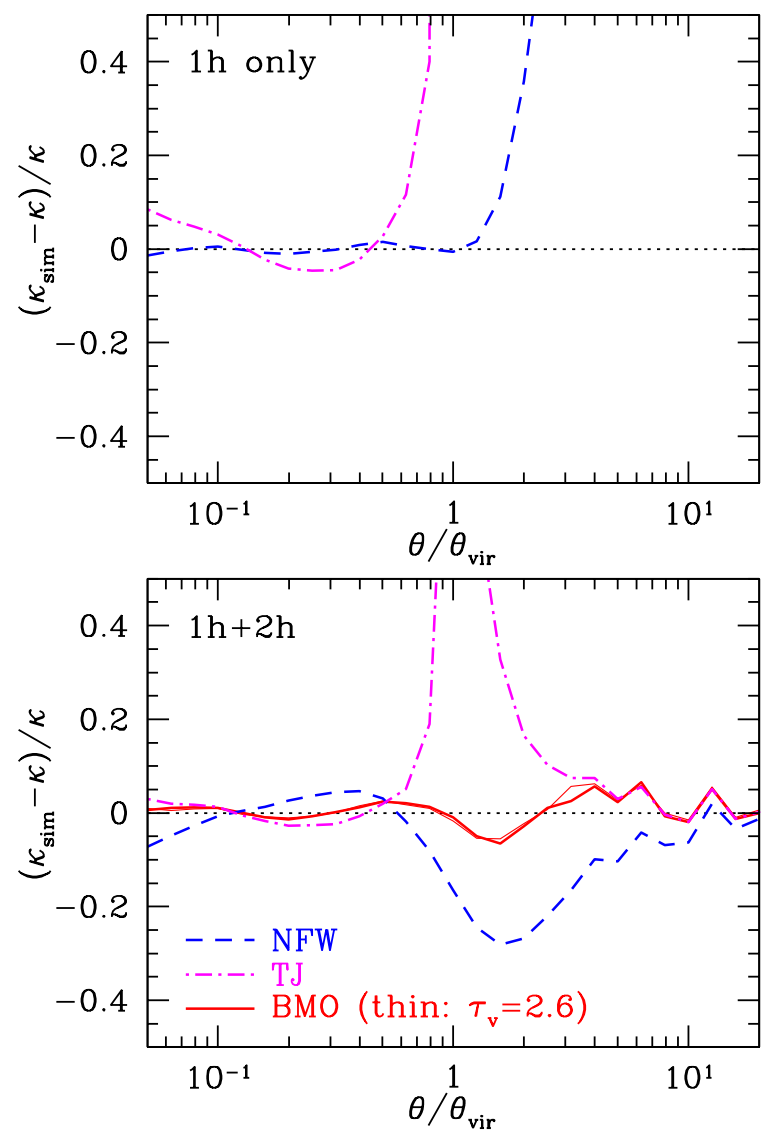

Figure 3. Residuals of convergence profile fitting (see Figure 2 for a representative example) averaged over all redshift and mass bins, plotted as a function of the normalised radius $\theta / \theta_{\text {vir }}$. Specifically we define the residual as $\left(\kappa_{\operatorname{sim}}-\kappa\right) / \kappa$, where $\kappa_{\operatorname{sim}}$ is the average convergence profile from ray-tracing simulations and $\kappa$ is the convergence profile of the best-fit analytic model. Lines are same as Figure 2 Upper: Residuals for fitting when only 1-halo term is considered (see also top panels of Figure2). Again, the result for the BMO is similar to the NFW result. Lower: Residuals when both 1-halo and 2-halo terms are included in the analytic model (see also bottom panels of Figure 2). The thin solid line indicates the result when the truncation radius in the BMO profile is fixed to $\tau_{v}=2.6$, the median value among fitting results for all redshift and mass bins (see Figure 1).

\section{IMPACT OF INACCURATE PROFILES ON CLUSTER WEAK LENSING STUDIES}

In most weak lensing studies, the NFW profile has been adopted in comparing with observed lensing signals, without including any contribution from the 2-halo term. However, the difference between assumed and true profiles can induce systematic biases in the interpretation of results. In this section, we investigate such systematic biases, assuming the analytic model calibrated by ray-tracing simulations (see Sec. 3) as a true cluster lensing profile.

\subsection{Shear profile fitting}

The most popular method to measure cluster masses from weak lensing data is to fit the tangential shear profile with analytic model predictions, for which the untruncated NFW

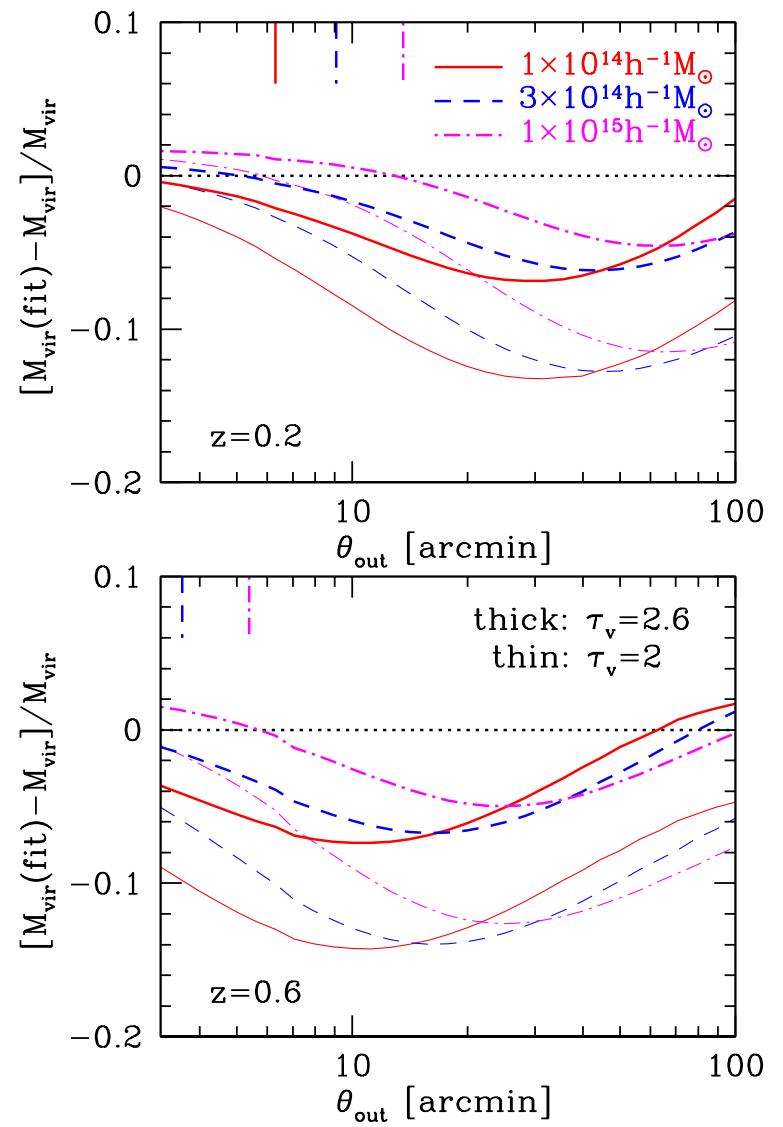

Figure 4. Biases in weak lensing mass estimates from shear profile fitting, as a function of the outermost fitting radius $\theta_{\text {out }}$, derived by adopting the BMO profile as the true lensing profile. The bias is defined as $\left[M_{\mathrm{vir}}(\mathrm{fit})-M_{\mathrm{vir}}\right] / M_{\mathrm{vir}}$, where $M_{\mathrm{vir}}$ is the input virial mass and $M_{\text {vir }}$ (fit) is the best-fit virial mass to the input reduced shear profile, using the NFW profile with no 2-halo term as a model for fitting. Note that the innermost fitting radius is fixed to $\theta_{\text {in }}=1^{\prime}$. We consider three input halo masses, $M_{\text {vir }}=10^{14} h^{-1} M_{\odot}($ solid $), 3 \times 10^{14} h^{-1} M_{\odot}($ dashed $)$, and $10^{15} h^{-1} M_{\odot}($ dash-dotted $)$. The halo redshifts are $z=0.2$ (upper) and 0.6 (lower). Thick and thin lines indicate results for the input truncation radii of $\tau_{v}=2.6$ and $\tau_{v}=2$, respectively. Vertical lines show virial radii for these cluster masses.

profile is usually adopted as the analytic model. Here we estimate how cluster masses derived by such shear profile fitting can be biased due to the deviation of the true cluster lensing profile from the one computed from the NFW profile.

In actual weak lensing analysis of observed data, we measure reduced shear profiles around clusters, which are defined by $g(\theta) \equiv \gamma_{\mathrm{T}}(\theta) /[1-\kappa(\theta)]$. For a given reduced shear profile, we usually derive the best-fit mass by minimizing the following $\chi^{2}$ :

$\chi^{2}=\sum_{i} \frac{\left[g\left(\theta_{i}\right)-g_{\mathrm{NFW}}\left(\theta_{i} ; M_{\mathrm{vir}}, c_{\mathrm{vir}}\right)\right]^{2}}{\sigma_{i}^{2}}$,

where $g\left(\theta_{i}\right)$ is observed reduced shear profile at the radius $\theta=\theta_{i}$ and $g_{\mathrm{NFW}}\left(\theta_{i} ; M_{\mathrm{vir}}, c_{\mathrm{vir}}\right)$ is the corresponding analytic model prediction assuming the NFW profile with no 2-halo term. We compute $g\left(\theta_{i}\right)$ assuming the BMO profile which has been shown to best reproduce lensing profiles in ray- 


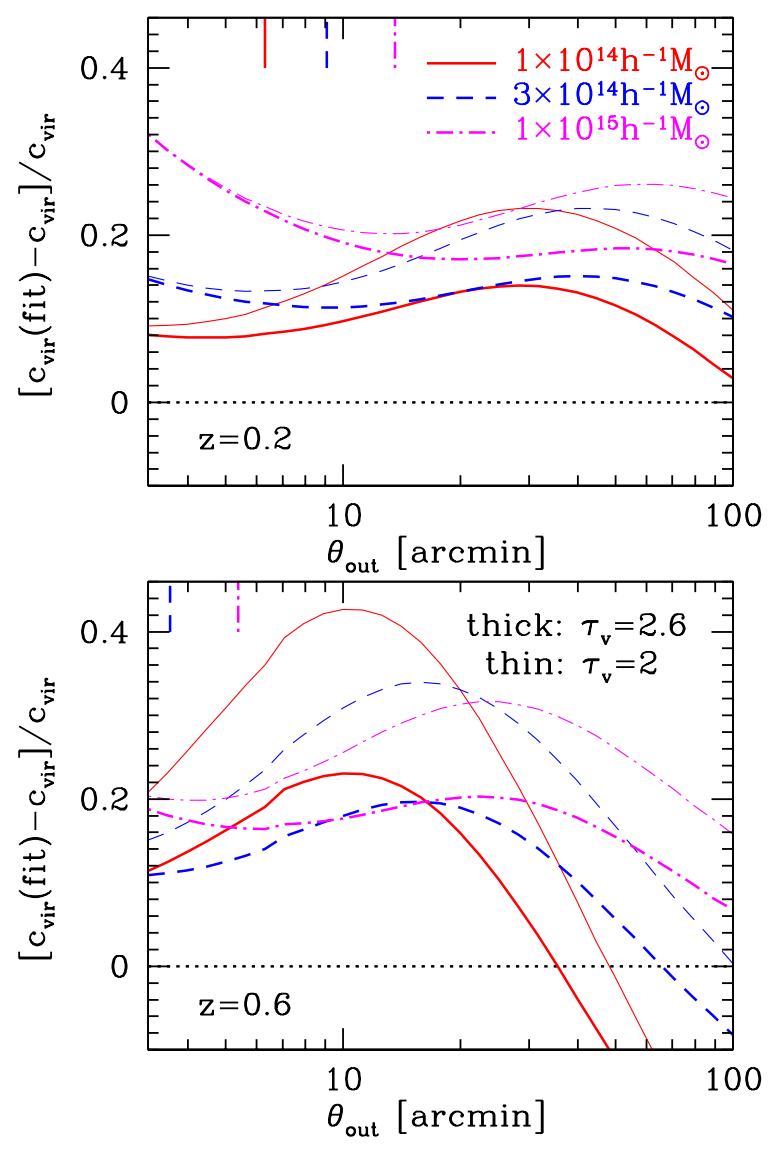

Figure 5. Same as Figure 4 but biases in estimates of the concentration parameter $c_{\mathrm{vir}}$ are shown.

tracing simulations (Sec. 31). Assuming the uniform source galaxy density, we adopt the error $\sigma_{i} \propto 1 / \theta_{i}$. The $\chi^{2}$ is computed in the range $\theta_{\text {in }}<\theta<\theta_{\text {out }}$ with an interval of $\Delta(\log \theta)=0.05$. We fix the innermost radius to $\theta_{\text {in }}=1^{\prime}$, and see how the best-fit mass $M_{\text {vir }}$ differs from the input mass as a function of $\theta_{\text {out }}$. In fitting, we vary both $M_{\text {vir }}$ and $c_{\text {vir }}$ to search for the best-fit parameter values.

Figure 4 shows the results, the biases as a function of $\theta_{\text {out }}$. We find that adopting a single NFW profile tends to underestimate virial masses for $\theta_{\text {out }} \sim 10^{\prime}-30^{\prime}$, a typical outermost radii adopted in the real analysis of cluster weak lensing data. The exact amount of the biases appears to be sensitive to $\tau_{v}$. For our canonical value of $\tau_{v}=2.6$, which was the median value among fitting results for many redshift and mass bins in ray-tracing simulations (see Figure 1) virial masses are underestimated by $\sim 5 \%$. On the other hand, if we adopt a slightly smaller value of $\tau_{v}=2$, virial masses can be underestimated by more than $10 \%$. If we choose very large values of $\theta_{\text {out }}$, the bias becomes smaller because of counteracting effects of the overpredciton of signals near the virial radius and the underprediction of the signals at very large radii where the 2-halo term contributions dominates. Our result suggests that we can reduce such mass estimation bias by restricting the fitting range small, $\theta_{\text {out }} \lesssim \theta_{\text {vir }}$.

Recently, Becker \& Kravtsov (2011) studied biases in weak lensing cluster mass estimates in details using a cosmological $N$-body simulations, and found that weak lens- ing masses are biased low by $\approx 5-10 \%$. They adopted $\theta_{\text {out }}=15^{\prime}, 20^{\prime}, 25^{\prime}$, and found a larger amount of the bias with increasing $\theta_{\text {out }}$. Thus our results are consistent with their results, both qualitatively and quantitatively, and moreover provide a physical explanation for their finding.

In Figure 5, we show biases in concentration parameter estimates. We find that the concentration parameter is generally overestimated, typically by $\sim 20 \%$. Clearly this is related to the mass estimation bias studied above. In lensing analysis with the NFW profile, there is a well-known degeneracy between mass and concentration parameter, and hence the underestimate of the mass has to be compensated by the increase of the concentration parameter in order to recover lensing signals near the cluster centre.

A caveat here is that the input $M_{\text {vir }}$ in the BMO profile differs from the true virial mass of the profile, which may make the interpretation of this result somewhat difficult. In fact we can avoid this problem by considering larger nonlinear overdensity $\Delta$, such as $\Delta \approx 1600$ used by Becker \& Kravtsov (2011), because the enclosed masses for the NFW and BMO profiles adopting the same $\rho_{s}$ should become more similar for larger values of $\Delta$. We confirm that our results shown in Figures 4 and 5] similarly hold for different nonlinear overdensities.

\subsection{Weak lensing mass peak}

Next we discuss how the uncertainty of lensing profiles can affect the interpretation and statistics of weak lensing mass peaks. The mass peak is usually characterised by the signalto-noise ratio:

$\nu=\frac{\kappa_{\text {peak }}}{\sigma_{\text {noise }}}$

where $\kappa_{\text {peak }}$ is the filtered peak amplitude computed by

$\kappa_{\text {peak }}=\int \gamma_{\mathrm{T}}(\theta) Q(\theta) 2 \pi \theta d \theta$,

with $Q(\theta)$ being the filter function. The noise $\sigma_{\text {noise }}$ is related to the intrinsic ellipticity and the number density of source galaxies, for which we assume $\sigma_{e}=0.35$ and $n_{\text {gal }}=30 \operatorname{arcmin}^{-2}$. In this paper, we consider two filter functions. One is the Gaussian filter Kaiser \& Squires 1993),

$Q(\theta)=\frac{1}{\pi \theta_{\text {in }}^{2}} \frac{1-\left(1+x^{2}\right) e^{-x^{2}}}{x^{2}}$,

and the other is a filter proposed by Hennawi \& Spergel (2005, hereafter HS05),

$$
\begin{aligned}
Q(\theta)= & \frac{1}{\pi \theta_{\text {in }}^{2}}\left[\frac{2 \log (1+x)}{x^{2}}-\frac{2}{x(1+x)}-\frac{1}{(1+x)^{2}}\right] \\
& \times e^{-x^{2} / 2 x_{\text {out }}^{2}}
\end{aligned}
$$

where $x \equiv \theta / \theta_{\text {in }}$ and $x_{\text {out }} \equiv \theta_{\text {out }} / \theta_{\text {in }}$. We adopt $\theta_{\text {in }}=1^{\prime}$ for the Gaussian filter, and $\theta_{\text {in }}=0.5^{\prime}$ and $\theta_{\text {out }}=10^{\prime}$ for the HS05 filter, which are typical values adopted in the literature.

We study the bias in $\nu$ assuming the true profile to be the BMO profile. Figure 6 shows how values of $\nu$ are biased by adopting the NFW profile with no 2-halo term. We find that the bias is $\lesssim 4 \%$ for peaks with $\nu \sim 3$ and smaller for 

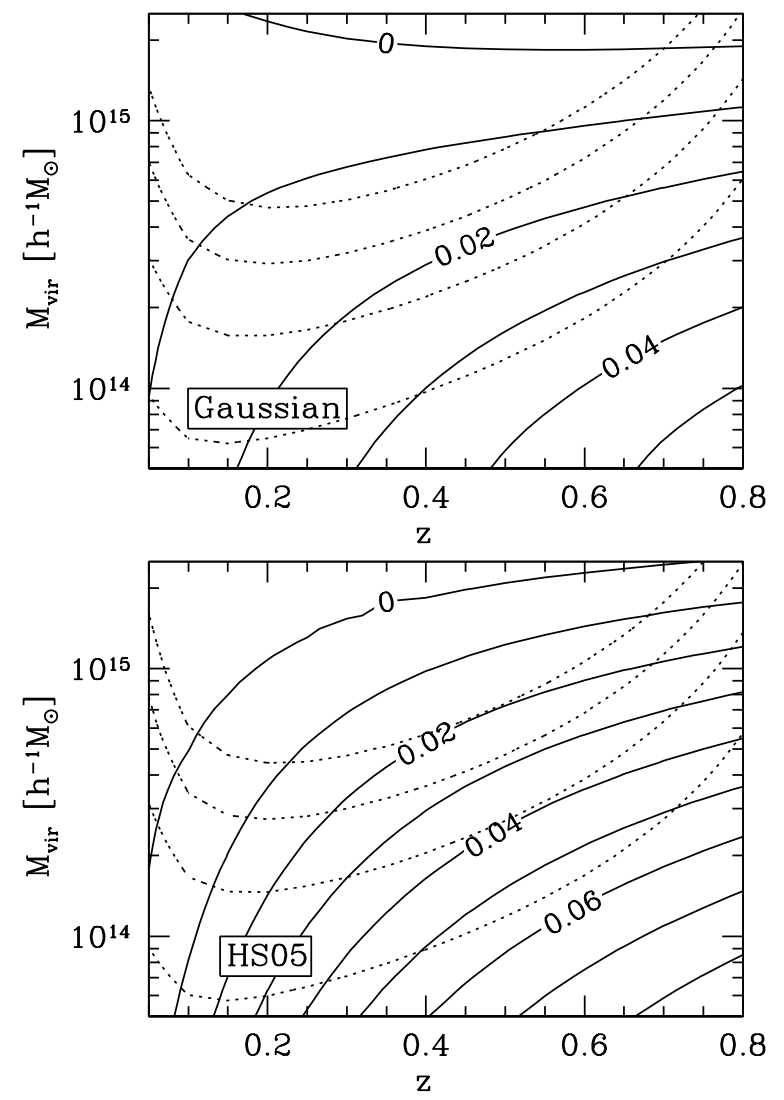

Figure 6. Contours of the biases in the signal-to-noise ratio $\nu$ defined in equation (15) in the mass-redshift plane. The bias is defined by $\left(\nu_{\mathrm{NFW}}-\nu\right) / \nu$, where $\nu_{\mathrm{NFW}}$ is the signal-to-noise ratio computed using the NFW profile with no 2-halo term, and $\nu$ is the true signal-to-noise ratio computed from the BMO profile with $\tau_{v}=2$. Note that the bias is even smaller for the canonical truncation radius of $\tau_{v}=2.6$. Dotted lines show contours for $\nu=3,5,7$, and 9, from bottom to top. The top panel is the result for the Gaussian filter (eq. 17), whereas the bottom panel is for the HS05 filter (eq. 18).

higher $\nu$. The bias is not so large because $\nu$ is mainly determined by the lensing profiles well inside the virial radius where the difference of the profiles are rather small. Note that the result shown in Figure 6 is for the truncation radius of $\tau_{v}=2$, which is smaller than our canonical value of $\tau_{v}=2.6$. The bias is even smaller if we adopt $\tau_{v}=2.6$.

In some previous work, the TJ profile has been used to compute $\nu$ instead of the NFW profile (e.g., Hamana et al. 2004). In Figure 7 we show the bias for the TJ profile. We confirm that the bias has a different sign from the case for the NFW profile, with a similar size of $\lesssim 4 \%$ for most of the parameter range we are interested in.

Therefore, in either case the effect of the outer profile on the calculation of the peak height $\nu$ is not very large, although it can be important for high-precision analytic predictions. We note that the result presented here is an averaged effect. In practice, there is a considerable scatter on the amount of correlated matter around clusters, which can affect the statistics of weak lensing mass peaks in various ways. The detailed study of statistical properties of weak lensing
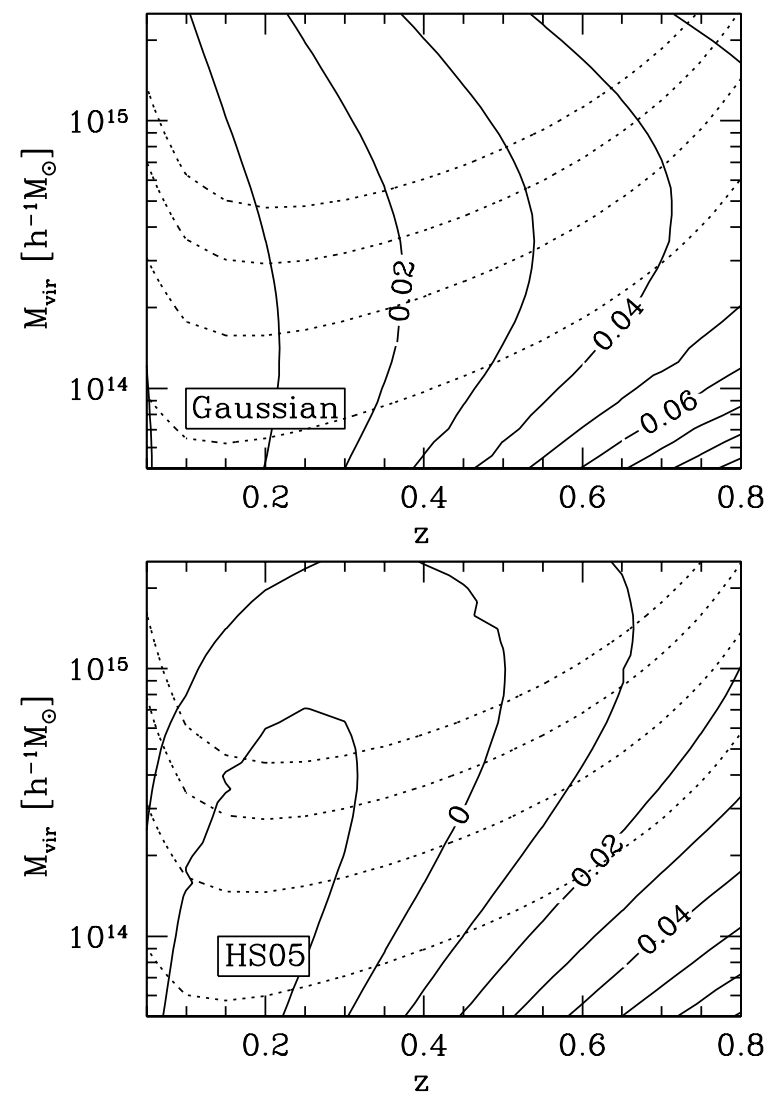

Figure 7. Same as Figure 6 but the biases for the TJ profile are shown.

mass peaks is currently underway, which will be reported in a forthcoming paper (Hamana \& Oguri, in preparation).

\section{SUMMARY AND DISCUSSIONS}

We have studied detailed lensing profiles and their impact on various cluster lensing studies. First, we compare our simple analytic model predictions, which consist of three different 1-halo terms and a 2-halo term, with a large set of ray-tracing in $N$-body simulations, with a particular emphasis on lensing profiles at around virial radii of massive haloes. Our ray-tracing solves the full light propagation from the source plane to the observer, and thus take accounts of all the matter fluctuations along the line-of-sight. We find that the BMO profile, which is essentially a smoothly truncated NFW profile, with the truncation radius of $\tau_{v} \sim 2-3$ with additional contribution from the 2-halo term best reproduces the lensing profiles in ray-tracing simulations. In contrast, the untruncated NFW profile tends to overpredict the convergence profiles by $20-30 \%$ at twice the virial radii when the 2-halo term is added. The TJ (hard-truncation) profile largely underpredicts the lensing signals at around the virial radii.

Next, assuming the BMO profile with the 2-halo term as the "true" cluster lensing profile, we have investigated biases in cluster weak lens studies coming from the adoption of the NFW profile as an analytic model. We find that, if observed reduced shear profiles are fitted by a single NFW compo- 
nent, as has commonly been done in cluster weak lensing analysis, we can underestimate cluster masses up to $5-10 \%$. The bias is most significant when the outer boundary for fitting is set to $\sim 10^{\prime}-30^{\prime}$ depending on the mass and redshift of the cluster, or equivalently several times the virial radius. We can reduce the bias by restricting the fitting range small with the outer boundary equal or smaller than the virial radius. On the other hand, the concentration parameter tends to be biased high (up to $\sim 20 \%$ or even higher) to compensate the underestimate of the mass. These results based on the analytic model appear to be consistent with recent numerical results by Becker \& Kravtsov (2011).

In the reduced shear fitting above, we have considered only intrinsic shapes of galaxies as a source of the error, i.e., ignored the contribution of the large-scale structure (cosmic shear) to the error (e.g., Hoekstra 2003; Dodelson 2004; Hoekstra et al. 2011). The error from the large-scale structure becomes increasingly important at larger radii, and thus decreases the relative weight of bins at large radii, implying that it reduces the bias in the mass and concentration parameter estimates. The exact amount of the reduction of the bias, however, depends on the source galaxy number density assumed. In real cluster weak lensing analysis, colour cuts have often been applied in order to minimise the dilution effect by cluster member galaxies, which significantly decreases source galaxy number densities used for the analysis. Because of this, the contribution of the large-scale structure to the total error budget tends to be subdominant in real data analysis (e.g., Oguri et al. 2010), but the large-scale structure should still contribute significantly at very large radii, e.g., $\theta \gtrsim 20^{\prime}$.

We have also examined the effect of the outer lensing profile on the signal-to-noise ratio $\nu$ of weak lensing mass peaks. We have considered two different filters, and found that $\nu$ can be biased by $\lesssim 4 \%$ for peaks with $\nu \gtrsim 3$ by adopting a single NFW component in calculating $\nu$. Although this level of the bias is not so large, it can be important in detailed comparisons of observed mass peaks with theory in the future.

While in this paper we have focused on biases due to modeling uncertainty of the outer lensing profiles, there are other effects that could also bias the weak lensing analysis. Among others, the halo triaxiality could have the most significant impact. Indeed, the large triaxiality of cluster-scale haloes suggests that the lensing signal is a strong function of the viewing angle (e.g., Oguri et al. 2005). We expect that the effect is almost averaged out by proper statistical analysis (e.g., Corless \& King 2009), but the exact amount of the residual bias due to the triaxiality should be sensitive to how the cluster sample is selected.

We note that the analytic model considered in the paper is subject to a potential improvement. For instance, the 2-halo term considered in the paper is accurate in the largescale limit. Near the cluster we may have to consider various effects such as non-linearity and stochasticity of the halo bias for more accurate theoretical prediction. In addition, for more robust separation of signals between the main halo and correlated matter, it is important to compare the three-dimensional matter distribution around clusters with projected lensing signals, which is left for future work.

Nevertheless, our analytic models presented in the paper, which has been tested against a large set of ray-tracing simulations, should be useful for various aspects of cluster weak lensing studies as a handy model of realistic lensing profiles in $N$-body simulations. In particular, we expect that they are invaluable in the stacked weak lensing study for which lensing signals can be detected much beyond the virial radius (e.g., Ménard et al. 2010; Umetsu et al. 2011). Given an important cosmological information coming from the 2halo term (e.g., Oguri \& Takada 2011), it is essential to have a proper analytic model which accurately predicted signals from the 1-halo to 2-halo terms, in order for an unbiased interpretation of stacked lensing data. The model is also useful for studying the effect of the outer profile uncertainty (including the contribution from correlated matter around clusters) on various cluster weak lensing analysis, as explicitly demonstrated in the paper for shear profile fitting and weak lensing mass peaks.

\section{ACKNOWLEDGMENTS}

We thank M. Becker, S. Masaki, M. Sato, M. Takada, and N. Yoshida for useful discussions. This work is supported in part by Grant-in-Aid for Scientific Research from the JSPS Promotion of Science (21740202).

\section{REFERENCES}

Baltz E. A., Marshall P., Oguri M., 2009, JCAP, 1, 15

Bartelmann M., 1996, A\&A, 313, 697

Becker M. R., Kravtsov A. V., 2011, arXiv:1011.1681

Broadhurst T., Umetsu K., Medezinski E., Oguri M., Rephaeli Y., 2008, ApJ, 685, L9

Cacciato M., van den Bosch F. C., More S., Li R., Mo H. J., Yang X., 2009, MNRAS, 394, 929

Corless V. L., King L. J., 2009, MNRAS, 396, 315

Dahle H., 2006, ApJ, 653, 954

de Putter R., White M., 2005, NewA, 10, 676

Dodelson S., 2004, PhRvD, 70, 023008

Duffy A. R., Schaye J., Kay S. T., Dalla Vecchia C., 2008, MNRAS, 390, L64

Eisenstein D. J., Hu W., 1998, ApJ, 496, 605

Fukushige T., Makino J., 2001, ApJ, 557, 533

Gavazzi R., Soucail G., 2007, A\&A, 462, 459

Geller M. J., Kurtz M. J., Dell'Antonio I. P., Ramella M., Fabricant D. G., 2010, ApJ, 709, 832

Hamana T., Takada M., Yoshida N., 2004, MNRAS, 350, 893

Hamana T., Miyazaki S., Kashikawa N., Ellis R. S., Massey

R. J., Refregier A., Taylor J. E., 2009, PASJ, 61, 833

Hayashi E., White S. D. M., 2008, MNRAS, 388, 2

Hennawi J. F., Spergel D. N., 2005, ApJ, 624, 59

Hilbert S., White S. D. M., 2010, MNRAS, 404, 486

Hoekstra H., 2003, MNRAS, 339, 1155

Hoekstra H., Hartlap J., Hilbert S., van Uitert E., 2011, MNRAS, in press (arXiv:1011.1084)

Hu W., Kravtsov A. V., 2003, ApJ, 584, 702

Johnston D. E., et al., 2007, arXiv, arXiv:0709.1159

Kaiser N., Squires G., 1993, ApJ, 404, 441

Kratochvil J. M., Haiman Z., May M., 2010, PhRvD, 81, 043519 
Kubo J. M., Khiabanian H., Dell'Antonio I. P., Wittman D., Tyson J. A., 2009, ApJ, 702, 980

Ma C.-P., Fry J. N., 2000, ApJ, 543, 503

Macciò A. V., Dutton A. A., van den Bosch F. C., 2008, MNRAS, 391, 1940

Mahdavi A., Hoekstra H., Babul A., Henry J. P., 2008, MNRAS, 384, 1567

Mandelbaum R., Tasitsiomi A., Seljak U., Kravtsov A. V.,

Wechsler R. H., 2005, MNRAS, 362, 1451

Mandelbaum R., Seljak U., 2007, JCAP, 6, 24

Mandelbaum R., Seljak U., Baldauf T., Smith R. E., 2010, MNRAS, 405, 2078

Mantz A., Allen S. W., Rapetti D., Ebeling H., 2010, MNRAS, 406, 1759

Marian L., Smith R. E., Bernstein G. M., 2010, ApJ, 709, 286

Masaki S., 2011, submitted to proceedings IDM 2010

Maturi M., Meneghetti M., Bartelmann M., Dolag K., Moscardini L., 2005, A\&A, 442, 851

Medezinski E., Broadhurst T., Umetsu K., Oguri M., Rephaeli Y., Benítez N., 2010, MNRAS, 405, 257

Ménard B., Scranton R., Fukugita M., Richards G., 2010, MNRAS, 405, 1025

Miyazaki S., et al., 2002, ApJ, 580, L97

Miyazaki S., Hamana T., Ellis R. S., Kashikawa N., Massey

R. J., Taylor J., Refregier A., 2007, ApJ, 669, 714

Nakamura T. T., Suto Y., 1997, PThPh, 97, 49

Navarro J. F., Frenk C. S., White S. D. M., 1997, ApJ, 490, 493

Oguri M., Takada M., Umetsu K., Broadhurst T., 2005, ApJ, 632, 841

Oguri M., et al., 2009, ApJ, 699, 1038

Oguri M., Takada M., Okabe N., Smith G. P., 2010, MNRAS, 405, 2215

Oguri M., Takada M., 2011, PhRvD, 83, 023008

Okabe N., Umetsu K., 2008, PASJ, 60, 345

Okabe N., Takada M., Umetsu K., Futamase T., Smith

G. P., 2010, PASJ, 62, 811

Pace F., Maturi M., Meneghetti M., Bartelmann M., Moscardini L., Dolag K., 2007, A\&A, 471, 731

Rozo E., et al., 2010, ApJ, 708, 645

Rozo E., Wu H.-Y., Schmidt F., 2011, arXiv:1009.0756

Sato M., Hamana T., Takahashi R., Takada M., Yoshida N., Matsubara T., Sugiyama N., 2009, ApJ, 701, 945

Schirmer M., Erben T., Hetterscheidt M., Schneider P., 2007, A\&A, 462, 875

Schneider P., 1996, MNRAS, 283, 837

Scoccimarro R., Sheth R. K., Hui L., Jain B., 2001, ApJ, 546,20

Seljak U., 2000, MNRAS, 318, 203

Sheldon E. S., et al., 2009, ApJ, 703, 2217

Takada M., Jain B., 2003a, MNRAS, 340, 580

Takada M., Jain B., 2003b, MNRAS, 344, 857

Tavio H., Cuesta A. J., Prada F., Klypin A. A., Sanchez-

Conde M. A., 2008, arXiv, arXiv:0807.3027

Tinker J., Kravtsov A. V., Klypin A., Abazajian K., Warren M., Yepes G., Gottlöber S., Holz D. E., 2008, ApJ, 688, 709

Tinker J. L., Robertson B. E., Kravtsov A. V., Klypin A., Warren M. S., Yepes G., Gottlöber S., 2010, ApJ, 724, 878

Umetsu K., et al., 2009, ApJ, 694, 1643
Umetsu K., Broadhurst T., Zitrin A., Medezinski E., Hsu L.-Y., 2011, ApJ, 729, 127

van Waerbeke L., 2000, MNRAS, 313, 524

Vikhlinin A., et al., 2009, ApJ, 692, 1060

White M., 2001, A\&A, 367, 27

Wright C. O., Brainerd T. G., 2000, ApJ, 534, 34

\section{APPENDIX A: EQUATIONS FOR LENSING BY THE BMO PROFILE}

The lensing properties of the BMO profile (eq. [7) were detailed in Baltz et al. (2009), in which analytic expressions of lens potential, deflection angle, convergence, etc. have been derived. Here we reproduce key equations for both $n=1$ and $n=2$ cases with the notation used in the paper.

For $n=1$, the convergence $\kappa(r)$ and average convergence $\bar{\kappa}(<r)$ are given by

$$
\begin{gathered}
\kappa(r)=\frac{4 \rho_{s} r_{s}}{\Sigma_{\text {crit }}} \frac{\tau^{2}}{2\left(\tau^{2}+1\right)^{2}}\left[\frac{\tau^{2}+1}{x^{2}-1}\{1-F(x)\}\right. \\
\left.+2 F(x)-\frac{\pi}{\sqrt{\tau^{2}+x^{2}}}+\frac{\tau^{2}-1}{\tau \sqrt{\tau^{2}+x^{2}}} L(x)\right], \\
\bar{\kappa}(<r)=\frac{4 \rho_{s} r_{s}}{\Sigma_{\text {crit }}} \frac{\tau^{2}}{\left(\tau^{2}+1\right)^{2} x^{2}}\left[\left(\tau^{2}+2 x^{2}+1\right) F(x)+\tau \pi\right. \\
\left.+\left(\tau^{2}-1\right) \ln \tau+\sqrt{\tau^{2}+x^{2}}\left\{-\pi+\frac{\tau^{2}-1}{\tau} L(x)\right\}\right],(\mathrm{A} 2)
\end{gathered}
$$

where $x \equiv r / r_{s}, \tau \equiv r_{t} / r_{s}=\tau_{v} c_{\mathrm{vir}}$, and $F(x)$ and $L(x)$ are

$F(x)= \begin{cases}\frac{1}{\sqrt{1-x^{2}}} \operatorname{arctanh} \sqrt{1-x^{2}} & (x<1), \\ \frac{1}{\sqrt{x^{2}-1}} \arctan \sqrt{x^{2}-1} & (x>1),\end{cases}$

$L(x)=\ln \left(\frac{x}{\sqrt{\tau^{2}+x^{2}}+\tau}\right)$.

It is also useful to show the three-dimensional enclosed mass $m_{\text {bmo }}(x)$ (see eq. 9]) for $n=1$. It is given by

$$
\begin{aligned}
& m_{\text {bmo }}(x)=\frac{\tau^{2}}{2\left(\tau^{2}+1\right)^{2}(1+x)} \\
& \quad \times\left[-2\left(\tau^{2}+1\right) x+4 \tau(x+1) \arctan (x / \tau)\right. \\
& \left.\quad+\left(\tau^{2}-1\right)(1+x) \ln \left(\tau^{2}(1+x)^{2} /\left(\tau^{2}+x^{2}\right)\right)\right] .
\end{aligned}
$$

Then the total mass $m_{\text {tot }}$ (see eq. 11) is

$$
\begin{aligned}
m_{\mathrm{tot}} & \equiv m_{\mathrm{bmo}}(\infty) \\
& =\frac{\tau^{2}}{\left(\tau^{2}+1\right)^{2}}\left[\pi \tau-\tau^{2}-1+\left(\tau^{2}-1\right) \ln \tau\right] .
\end{aligned}
$$

For $n=2, \kappa(r)$ and $\bar{\kappa}(<r)$ are given by

$$
\begin{aligned}
& \kappa(r)=\frac{4 \rho_{s} r_{s}}{\Sigma_{\text {crit }}} \frac{\tau^{4}}{4\left(\tau^{2}+1\right)^{3}}\left[\frac{2\left(\tau^{2}+1\right)}{x^{2}-1}\{1-F(x)\}\right. \\
&+8 F(x)+\frac{\tau^{4}-1}{\tau^{2}\left(\tau^{2}+x^{2}\right)}-\frac{\pi\left[4\left(\tau^{2}+x^{2}\right)+\tau^{2}+1\right]}{\left(\tau^{2}+x^{2}\right)^{3 / 2}}
\end{aligned}
$$

(C) RAS, MNRAS 000, 111 


$$
\begin{aligned}
+ & \left.\frac{\tau^{2}\left(\tau^{4}-1\right)+\left(\tau^{2}+x^{2}\right)\left(3 \tau^{4}-6 \tau^{2}-1\right)}{\tau^{3}\left(\tau^{2}+x^{2}\right)^{3 / 2}} L(x)\right] \\
\bar{\kappa}(<r) & =\frac{4 \rho_{s} r_{s}}{\Sigma_{\text {crit }}} \frac{\tau^{4}}{2\left(\tau^{2}+1\right)^{3} x^{2}}\left[2\left(\tau^{2}+4 x^{2}-3\right) F(x)\right. \\
& +\frac{1}{\tau}\left\{\pi\left(3 \tau^{2}-1\right)+2 \tau\left(\tau^{2}-3\right) \ln \tau\right\} \\
& +\frac{1}{\tau^{3} \sqrt{\tau^{2}+x^{2}}}\left\{-\tau^{3} \pi\left(4 x^{2}+3 \tau^{2}-1\right)+\right. \\
& \left.\left.+\left[2 \tau^{4}\left(\tau^{2}-3\right)+x^{2}\left(3 \tau^{4}-6 \tau^{2}-1\right)\right] L(x)\right\}\right]
\end{aligned}
$$

The three-dimensional enclosed mass $m_{\mathrm{bmo}}(x)$ and the total mass $m_{\text {tot }}$ for $n=2$ were already given in equations (10) and (12), respectively.

From the above expressions, the tangential shear $\gamma_{\mathrm{T}}(r)$ and deflection angle $\phi_{r}(r)$ can be computed as

$\gamma_{\mathrm{T}}(r)=\bar{\kappa}(<r)-\kappa(r)$,

$\phi_{r}(r)=r \bar{\kappa}(<r)$.

\section{APPENDIX B: FOURIER TRANSFORM OF THE BMO PROFILE}

The Fourier transform of a dark halo density profile constitute an important ingredient of the so-called halo model approach (e.g., Seliak 2000; Ma \& Frv 2000; Scoccimarro et al. 2001). In this appendix we study the Fourier transform of the BMO profile.

We consider the three-dimensional Fourier transform of $u_{M}(r) \equiv \rho(r) / M_{\text {vir }}, \tilde{u}_{M}(k)$ with $k$ being the comoving wavenumber. We note that the Fourier transform of lensing signals (convergence and tangential shear profiles) can also described by using $\tilde{u}_{M}(k)$ as a consequence of the projection-slice theorem (see Oguri \& Takada 2011). We find that $\tilde{u}_{M}(k)$ for the BMO profile can analytically be expressed as

$$
\begin{aligned}
\tilde{u}_{M}(k) & =\frac{\tau}{2 m_{\mathrm{nfw}}\left(c_{\mathrm{vir}}\right)\left(1+\tau^{2}\right)^{2} x} \\
\times & {\left[2\left(\tau^{2}-1\right) P(\tau x)-2 \tau \pi e^{-\tau x}\right.} \\
& +\tau\{\pi-2 \operatorname{Si}(x)\}\left\{2 \cos x+\left(\tau^{2}+1\right) x \sin x\right\} \\
& \left.+2 \tau \operatorname{Ci}(x)\left\{2 \sin x-\left(\tau^{2}+1\right) x \cos x\right\}\right],
\end{aligned}
$$

for $n=1$, and

$$
\begin{aligned}
\tilde{u}_{M}(k) & =\frac{\tau}{4 m_{\mathrm{nfw}}\left(c_{\mathrm{vir}}\right)\left(1+\tau^{2}\right)^{3} x} \\
\times & {\left[2\left(3 \tau^{4}-6 \tau^{2}-1\right) P(\tau x)-2 \tau\left(\tau^{4}-1\right) x Q(\tau x)\right.} \\
& -2 \tau^{2} \pi e^{-\tau x}\left\{\left(\tau^{2}+1\right) x+4 \tau\right\} \\
& +2 \tau^{3}\{\pi-2 \operatorname{Si}(x)\}\left\{4 \cos x+\left(\tau^{2}+1\right) x \sin x\right\} \\
& \left.+4 \tau^{3} \operatorname{Ci}(x)\left\{4 \sin x-\left(\tau^{2}+1\right) x \cos x\right\}\right],
\end{aligned}
$$

for $n=2$, where $x \equiv(1+z) k r_{s}$. Note that $m_{\mathrm{nfw}}\left(c_{\mathrm{vir}}\right)$ in the denominator originates from the division of the density profile by $M_{\mathrm{vir}}$; if instead the profile is normalised by the total mass $M_{\text {tot }}, m_{\mathrm{nfw}}\left(c_{\mathrm{vir}}\right)$ should be replaced with $m_{\text {tot }}$. The functions $P(x)$ and $Q(x)$ are defined as

$$
\begin{aligned}
& P(x)=\sinh (x) \operatorname{Chi}(x)-\cosh (x) \operatorname{Shi}(x), \\
& Q(x)=\frac{d P}{d x}=\cosh (x) \operatorname{Chi}(x)-\sinh (x) \operatorname{Shi}(x) .
\end{aligned}
$$

For reference, the sine and cosine integrals and the hyperbolic sine and cosine integrals are given by

$$
\begin{aligned}
\operatorname{Si}(x) & \equiv \int_{0}^{x} \frac{\sin t}{t} d t \\
\operatorname{Ci}(x) & \equiv \gamma+\ln x+\int_{0}^{x} \frac{\cos t-1}{t} d t \\
\operatorname{Shi}(x) & \equiv \int_{0}^{x} \frac{\sinh t}{t} d t, \\
\operatorname{Chi}(x) & \equiv \gamma+\ln x+\int_{0}^{x} \frac{\cosh t-1}{t} d t,
\end{aligned}
$$

with $\gamma=0.57721566 \ldots$ being the Euler-Mascheroni constant. While $P(x)$ and $Q(x)$ can easily be computed numerically (except at large $x$ where the round-off error dominates), we provide fitting formulae for them:

$$
\begin{aligned}
P_{\text {fit }}(x)= & -\left[\frac{1}{x}+\frac{b x^{e}}{c+(x-d)^{2}}\right]\left(\frac{x^{4}}{x^{4}+a^{4}}\right)^{f} \\
& +x(\gamma+\ln x-1)\left(\frac{a^{4}}{x^{4}+a^{4}}\right)^{f},
\end{aligned}
$$

with $(a, b, c, d, e, f)=(1.5652,3.38723,6.34891,0.817677$, $-0.0895584,0.877375)$, and

$$
\begin{aligned}
Q_{\text {fit }}(x) & =\left[\frac{1}{x^{2}}+\frac{b x^{e}}{c+(x-d)^{4}}\right]\left(\frac{x^{4}}{x^{4}+a^{4}}\right)^{g} \\
+ & {\left[(\gamma+\ln x)\left(1+\frac{x^{2}}{2}\right)-\frac{3}{4} x^{2}\right]\left(\frac{a^{4}}{x^{4}+a^{4}}\right)^{f}, }
\end{aligned}
$$

with $(a, b, c, d, e, f, g)=(2.26901,-2839.04,265.511$, $-1.12459,-2.90136,1.86475,1.52197)$. These fitting formulae are obtained simply by connecting series expansions at $x=0$ and $\infty$. We find that $P_{\text {fit }}(x)$ is accurate at better than $0.5 \%$ for any positive values of $x$. The function $Q_{\mathrm{fit}}(x)$ is also accurate at better than $0.5 \%$, except at around $x \approx 0.88$ where $Q(x)$ has a root (i.e., $Q(x) \approx 0$ ). 